\title{
Disposable cartridge biosensor platform for portable diagnostics
}

Yusuf S. Yaras, Onur Cakmak, Ali B. Gunduz, Gokhan Saglam, Selim Olcer, et al.

Yusuf S. Yaras, Onur Cakmak, Ali B. Gunduz, Gokhan Saglam, Selim Olcer, Aref Mostafazadeh, Ibrahim Baris, Fehmi Civitci, Goksen G. Yaralioglu, Hakan Urey, "Disposable cartridge biosensor platform for portable diagnostics," Proc. SPIE 10055, Optics and Biophotonics in Low-Resource Settings III, 1005500 (3 March 2017); doi: 10.1117/12.2254729 


\title{
Disposable cartridge biosensor platform for portable diagnostics
}

\author{
Yusuf S. Yaras ${ }^{\mathrm{a}}$, Onur Cakmak ${ }^{\mathrm{b}}$, Ali B. Gunduz ${ }^{\mathrm{a}}$, Gokhan Saglam ${ }^{\mathrm{a}}$, Selim Olcer ${ }^{\mathrm{a}}$, Aref \\ Mostafazadeh $^{\mathrm{a}}$, Ibrahim Baris ${ }^{\mathrm{a}}$, Fehmi Civitci ${ }^{\mathrm{c}}$, Goksen G. Yaralioglu ${ }^{\mathrm{d}}$, Hakan Urey*a \\ ${ }^{\mathrm{a}}$ Koc University, Rumelifeneri Yolu, Sariyer, Istanbul, Turkey 34450; ${ }^{\mathrm{b}}$ Columbia University, New \\ York, NY, 10027, USA, ' Istanbul Technical University, Ayazağa Campus, Maslak, Istanbul, Turkey \\ 34469; ${ }^{~}$ Özyeğin University, Nisantepe District, Orman Street, Cekmekoy, Istanbul, Turkey 34794
}

Keywords: coagulation, biosensor, optical read out, MEMS, fiber sensor, aPTT, point of care

\begin{abstract}
We developed two types of cantilever-based biosensors for portable diagnostics applications. One sensor is based on MEMS cantilever chip mounted in a microfluidic channel and the other sensor is based on a movable optical fiber placed across a microfluidic channel. Both types of sensors were aimed at direct mechanical measurement of coagulation time in a disposable cartridge using plasma or whole blood samples. There are several similarities and also some important differences between the MEMS based and the optical fiber based solutions. The aim of this paper is to provide a comparison between the two solutions and the results. For both types of sensors, actuation of the cantilever or the moving fiber is achieved using an electro coil and the readout is optical. Since both the actuation and sensing are remote, no electrical connections are required for the cartridge. Therefore it is possible to build low cost disposable cartridges. The reader unit for the cartridge contains light sources, photodetectors, the electro coil, a heater, analog electronics, and a microprocessor. The reader unit has different optical interfaces for the cartridges that have MEMS cantilevers and moving fibers. MEMS based platform has better sensitivity but optomechanical alignment is a challenge and measurements with whole blood were not possible due to high scattering of light by the red blood cells. Fiber sensor based platform has relaxed optomechanical tolerances, ease of manufacturing, and it allows measurements in whole blood. Both sensors were tested using control plasma samples for activated-Partial-Thromboplastin-Time (aPTT) measurements. Control plasma test results matched with the manufacturer's datasheet. Optical fiber based system was tested for aPTT tests with human whole blood samples and the proposed platform provided repeatable test results making the system method of choice for portable diagnostics.
\end{abstract}

\section{INTRODUCTION}

Blood coagulation time measurement is essential part of both diagnostics and therapeutics ${ }^{1}$. Coagulation tests are required for patient condition evaluation before any surgical operation ${ }^{2}$. They are also used to assess the hepatic and renal disorders ${ }^{1}$ or dengue hemorrhagic fever ${ }^{3}$. Patients receiving any anticoagulant treatment ${ }^{4}$ and patients with or risk of cardiac problems such as embolism, atrial fibrillation or stroke need to undergo blood coagulation tests periodically ${ }^{5}$. Dosage of anticoagulants must be adjusted according to blood coagulation test results. Over 800 million coagulation tests are performed annually mostly in hospitals and clinical laboratories for emergency situations, surgical operations, as well as periodic monitoring of certain patients ${ }^{6}$. Thus it is crucial to develop reliable and fast Point-of-Care (PoC) coagulation assays for low resource settings. A good PoC coagulation assay should have several key elements: the tests should be reliable and cost effective. Moreover, the tests should generate results with very small volumes of whole blood samples ${ }^{7}$ as low as couple of microliters.

In clinical settings, there are two standard approaches for coagulation time measurements: mechanical and optical ${ }^{7}$. Optical sensing tracks changes in optical properties of plasma as it coagulates. This method requires plasma separation from whole blood due to the high absorption in whole blood ${ }^{8}$. The second standard clinical method is mechanical. In this method a sharp increase in viscosity is observed due to fibrin formation during coagulation. The second approach is called direct mechanical measurement and it is usually preferred over optical methods. However, both methods are suitable to work with plasma. The plasma is extracted from the whole blood samples by centrifuging. Conducting

Optics and Biophotonics in Low-Resource Settings III, edited by David Levitz, Aydogan Ozcan, David Erickson, Proc. of SPIE Vol. 10055, 1005500 - (C) 2017 SPIE · CCC code: 1605-7422/17/\$18 · doi: 10.1117/12.2254729 
coagulation tests on plasma complicates coagulation measurement protocol since plasma extraction requires a dedicated tool, extra time and larger blood sample and this reduces the usability of both approaches for PoC applications.

On the other hand, electro chemical ${ }^{7}$ measurement based technologies are extensively used in $\mathrm{PoC}$ applications since these technologies enable simple sensor designs. Coagulation time can be measured by observing electrical resistance of the blood since the ion composition of the blood changes as it coagulates. However chemical coatings in the cartridges must be kept consistent for reliable test results. Moreover, since it is an indirect measurement method, multiple drug usage and certain health conditions may affect the reliability of the results ${ }^{9}$.

In our group, we developed a biosensor platform for low cost PoC coagulation time measurements. The measurement is based on observing the vibrations of a mechanical structure. A cantilever beam submersed in the blood sample is vibrated around its resonant frequency similar to direct mechanical testing which is considered golden standard. As blood coagulates, viscosity of the blood increases hence resonance characteristics of the cantilever changes. Two different types of sensors were developed using the same biosensor platform: one sensor utilizes MEMS cantilevers whereas the other sensor utilizes optical fiber as a mechanical cantilever. Actuation of the cantilevers is carried out remotely by an electro coil and read out is optical for both sensors. The remote nature of the actuation and the read-out provides tremendous advantage towards building a system with independent reader unit and disposable cartridges.

\section{METHODOLOGY}

\subsection{Working principle}

The resonant frequency and the quality factor of the cantilever-like systems, operating in viscous liquid media, is affected by the viscosity of the liquid ${ }^{10,11}$. When the coagulation is triggered, first thrombin acts on fibrinogen and results in fibrin generation; those generated fibrins aggregate and form insoluble clots. This unique cascade and the formation of the clots cause a sudden change in blood viscosity ${ }^{12}$. Both the MEMS based and the fiber-optic based techniques make use of this phenomenon. The viscosity change creates an increase in the hydrodynamic loading of the vibrating structures in viscous liquids according to Sader's theory ${ }^{10,11}$. We observe the effect of viscosity change by monitoring the phase shift between the drive signal and the phase of the mechanical displacement. We excite the nickel cantilever or the fiber at a certain frequency around the resonant peak. During the coagulation the phase difference at the excitation frequency changes and creates a unique signature. The coagulation time can be determined from this unique signature.

\subsection{MEMS based sensor}
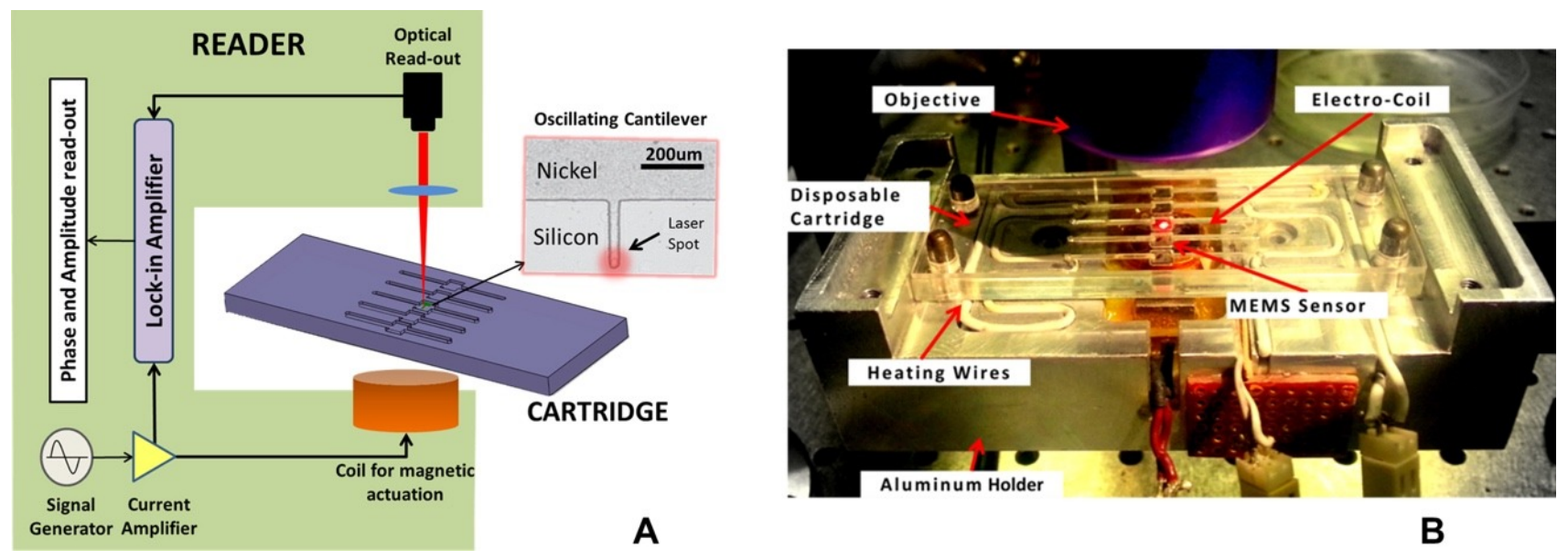

A

B

Figure 1. (A) Schematic view of the MEMS based measurement setup. (B) A photo of the measurement setup ${ }^{12}$

The MEMS Sensor fabrication was done similar to the procedure that we reported in our previous works ${ }^{13}$. Briefly, a simple one mask procedure consisting of sputter, photolithography, electrodeposition and wet etching steps was performed to fabricate nickel cantilevers. The dimensions of the cantilevers are $200 \mu \mathrm{m} \times 20 \mu \mathrm{m} \times 2 \mu \mathrm{m}$. These 
dimensions were selected based on the operating frequency of the lock-in amplifier used in the experiments. After the fabrication, the MEMS dies were placed inside the PMMA microfluidic cartridge. They were fixed inside the micromachined channels of the cartridge by UV cured epoxy (Norland Optical Adhesive 81). The microfluidic channels had $200 \mu \mathrm{m}$ depth and $1 \mathrm{~mm}$ width. Five channels were patterned on the microfluidic cartridge as shown in Fig. 1 and they can be functionalized for different tests. The same UV curable epoxy had also been used for sealing the parallel microfluidic channels from each other. The volume of each channel is $10 \mu \mathrm{l}$ which can be further reduced with optimized cartridge geometry.

The schematic of the experimental setup and the principle of operation were demonstrated in Fig.1a. An external coil, placed under the PMMA cartridge is used for the actuation of the nickel cantilevers. The mechanical vibrations of the cantilevers are monitored using an optical read-out. Both magnetic actuation and optical read-out is achieved remotely without any electrical contacts to the cartridge. The MEMS sensor results presented in this study were obtained with a Laser Doppler Vibrometer (LDV) which can be replaced with other techniques such as grating interferometer or knifeedge methods which have been successfully implemented by our group, previously ${ }^{14}$. A lock-in amplifier (Zurich Instruments HF2LI) is used to continuously monitor the phase difference between the coil drive and the LDV. This phase difference recorded simultaneously using a LabVIEW interface during the experiments. All experiments were conducted at $37 \pm 0.1^{\circ} \mathrm{C}$.

In addition to the above mentioned optical read-out methods, optical lever method with pickup fibers can also be used ${ }^{15}$. Fig 2 shows the general principle of the system. In this method, equally spaced laser beams (generated by a diffraction grating) were focused to the tip of the cantilevers using a lens. Reflected beams from the cantilever tips were relayed to photodiodes using a custom made optical fiber array pick-up head. All the cantilevers were driven at a fixed frequency sine wave near their resonances through the electro-coil. The cantilever vibration affects the direction of the reflected laser beam, therefore it modulates the light coupled into the pickup fiber. The other ends of the pick-up fibers are coupled to separate photodetectors for each fiber. Using optical fibers to relay light from cantilevers to photodetectors helps accommodating various cantilever pitches without constructing complex photodetector arrays. Another important advantage of using optical fibers is electrical noise immunity. This approach allows placement of detection electronics further away from the electro coil which may potentially create interference signal on the high gain detection electronics.

For a more precise estimation of the spot size on the focal plane and also to study the clipping effects, optical design was simulated on the Zemax software using physical wave propagation method. Cantilevers were modeled as rectangular mirrors. Although, the maximum displacement occurs at the tip of the cantilever, the laser spot has finite focal size and it must be aligned on the cantilever beam at a certain distance from the tip to keep the clipping of the spot at acceptable levels. With available optical elements, minimum achievable focus diameter was determined to be $28 \mu \mathrm{m}$ on the cantilevers. Practically, the best sensitivity results were observed when the center of the laser spot is placed $25 \mu \mathrm{m}$ away from the tip for our $200 \mu \mathrm{m}$ cantilevers. Fig. $2 \mathrm{~b}$, shows the reflected beam profile for $200 \mu \mathrm{m}$ length cantilevers with different widths of $20 \mu \mathrm{m}, 30 \mu \mathrm{m}, 50 \mu \mathrm{m}$ and $100 \mu \mathrm{m}$. Fig. 2a also points out a difficulty in aligning lasers with the cantilevers when the cantilevers are initially tilted. With two cantilevers, it is always possible to align the optical fiber head for optimum beam locations by rotating the optical head. Fig. 2a shows a sketch of shifted beams from bended cantilevers when both cantilevers have different tilts and the rotated optical head to capture the optimum signal. With more than two cantilevers, the alignment for capturing maximum signal from all the cantilevers was challenging.

The performance of MEMS based sensor was investigated for standard coagulation tests with blood plasma. The results of these tests are demonstrated and discussed in the results section. The MEMS based system provides high resolution and capability of conducting PT tests which allow very limited preparation times. On the other hand, the Signal-to-Noise Ratio (SNR) significantly drops for the measurements in whole blood due to scattering from red blood cells. One other technical challenge was the tight alignment tolerances for the cantilevers in parallel channels. Since the cantilevers' width is only $20 \mu \mathrm{m}$, the laser spot should be focused to that small region when the cartridge is placed in the reader unit. This results in a very stringent placement requirement between the cartridge and the optical read out system. The optical fiber based sensor platform relaxes the tight positioning requirement as well as it works in whole blood with high SNR. 

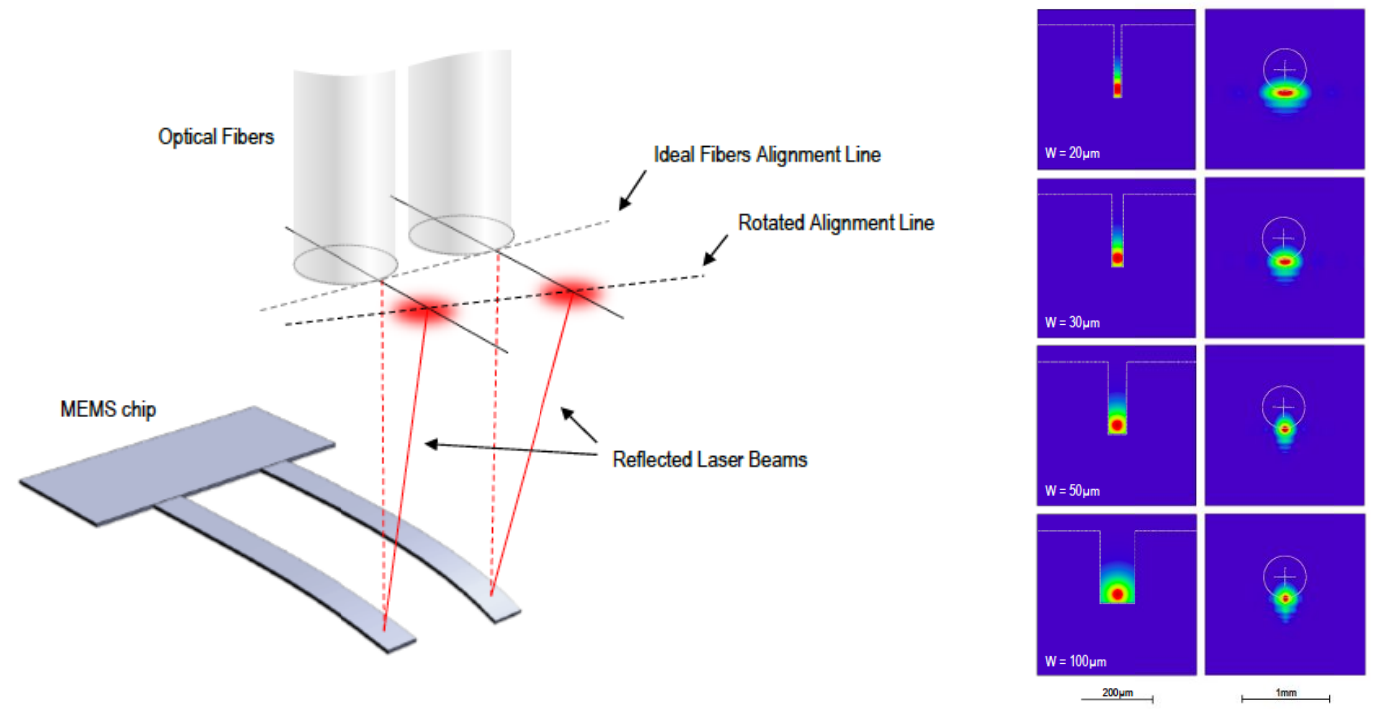

Figure 2. (A) Optical alignment of two channel optical readout with bended cantilevers (B) Profile of the laser beam on the cantilever and the reflected beam on the fiber plane for $200 \mu \mathrm{m}$ length cantilevers with different widths of $20 \mu \mathrm{m}, 30$ $\mu \mathrm{m}, 50 \mu \mathrm{m}$ and $100 \mu \mathrm{m}$. Dashed lines shows the edges of the cantilever and the location of the large core optical fiber (Ø $=486 \mu \mathrm{m})$.

\subsection{Optical fiber based sensor}

Fiber based sensor utilizes two optical fibers: one movable fiber used as vibrating cantilever and one fixed fiber as the pick-up fiber. Similar to MEMS based sensor, movable fiber is immersed in the plasma or whole blood sample and vibrated around its resonant frequency by an external electro coil. A high purity nickel piece is attached to the movable fiber to facilitate remote magnetic actuation. Pick up fiber is placed in line with the vibrating fiber. When movable fiber is vibrated, light coupled from moving fiber to pick up fiber is modulated. This modulated light is collected by the photo detector. Fig. 3 shows the fiber based sensor schematic. Vibrating fiber is suspended in the microfluidic channel and immersed in the blood sample. Note that fibers and microfluidic channels are placed inside disposable cartridge whereas light source, detector, external electromagnetic coil and electronics are placed in the reader unit.

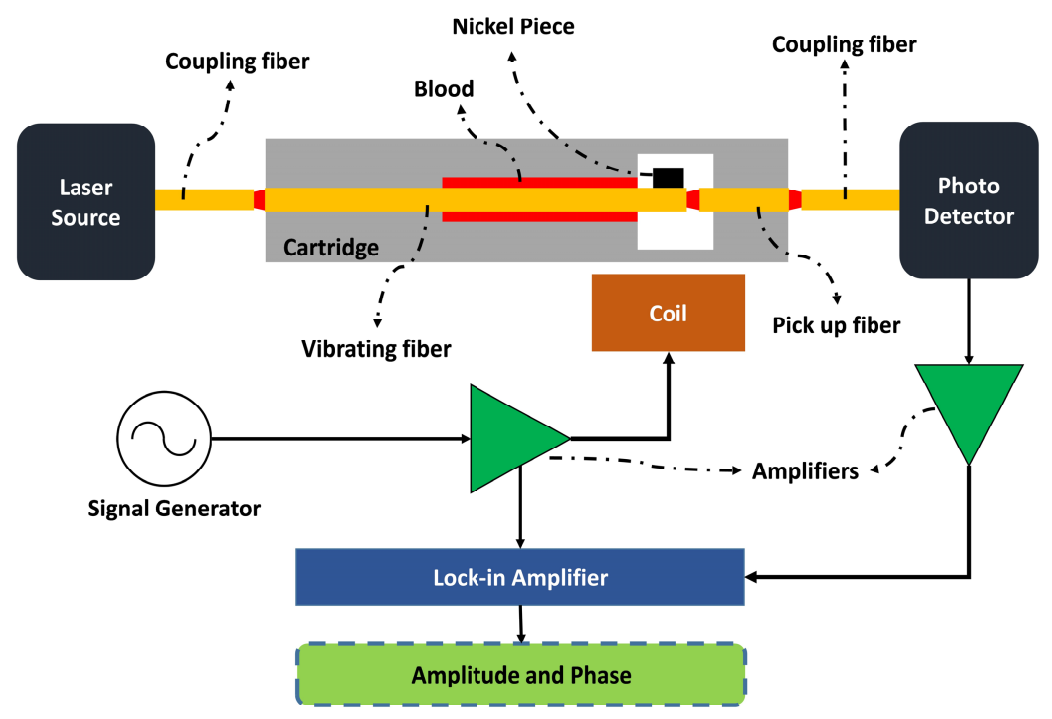

Figure 3. Schematic view of the optical fiber based measurement setup. 
Alignment tolerance between the disposable cartridge and the reader unit is curial for mass production. Alignment tolerance for MEMS based sensor was less than $20 \mu \mathrm{m}$. For the MEMS sensor based system, small alignment errors can easily result in laser beams to miss the MEMS cantilevers. For the fiber sensor based system, we analyzed the alignment tolerances using Zemax software and physical wave propagation method. Misalignment was introduced in one direction. Note that, light is coupled from the side of the cartridge and guided through the fibers whereas in MEMS based sensor light is projected onto the cantilever beams and the reflected light is picked up. Thus, MEMS based sensor is sensitive to cantilever tilts and position whereas optical fiber based sensor is only sensitive to position of the sensor with respect to coupling fibers. Sensitivity for the misalignment was further investigated with experimental test. Fig. 4 shows the normalized coupled power with respect to misalignment between coupling fiber and sensor. Coupled power of the light is halved when misalignment exceeds $200 \mu \mathrm{m}$.

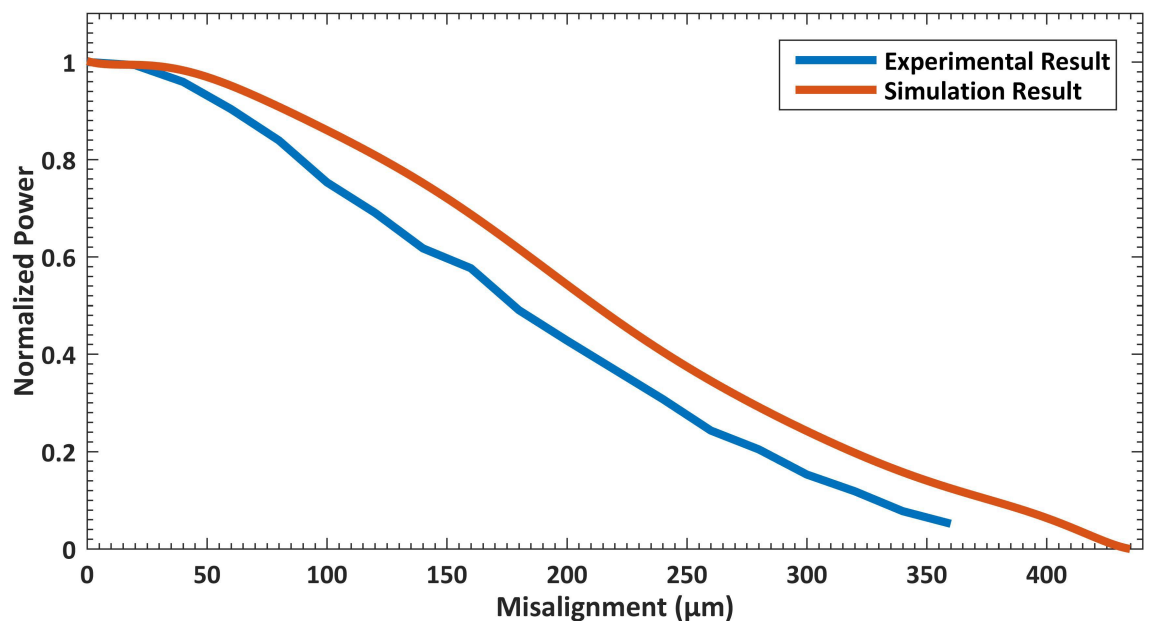

Figure 4. Normalized power of the light coupled between sensor and reader with respect to optical misalignment. Gap between fibers is $250 \mu \mathrm{m}$, core diameter is $400 \mu \mathrm{m}$ and cladding diameter is $440 \mu \mathrm{m}$.

The disposable cartridges were made of Poly Methyl Methacrylate (PMMA). Microfluidic channels and fiber grooves were machined into PMMA parts. Relatively large optical fibers were selected as a $400 \mu \mathrm{m}$ core and $440 \mu \mathrm{m}$ cladding diameter to relax the alignment requirements between the reader unit and the cartridge. As simulated above, this configuration gives around 200 um alignment tolerance of the disposable cartridge to reader unit. $200 \mu \mathrm{m}$ of alignment tolerance is relatively achievable with conventional machining techniques enabling repeatable and reliable operation. A high purity nickel piece is glued close to the free end of the vibrating fiber. Optical fibers and PMMA parts are bonded together using UV curable epoxy (NOA 81, Norland Products, US). Note that whole sensor is fabricated using off the shelf components and conventional fabrication techniques, clean room is not needed for the fabrication. PMMA machining can be easily replaced with molding which is cheaper and faster in mass production. Therefore, disposable cartridges can be manufactured cost effectively in large numbers.

\subsection{Reader unit}

We have tested various optical read out schemas for MEMS based sensors. Initially we used a commercial Laser Doppler Vibrometer (LDV). LDV based read out scheme offers high SNR, however cost, large volume and the complexity of the LDV make the biosensor platform not suitable for PoC applications. Then we used an integrated diffraction grating to measure the cantilever vibration. In this method, the light reflecting form the cantilever and the substrate create an interference pattern ${ }^{14}$. This leads to an intensity modulation on the photodiode in response to the deflection of Ni cantilever with respect to the Si reference surface. Cantilever movement was tracked by a photo detector capturing the first diffracted order. Small size of the cantilevers and oblique light paths require very tight alignment tolerances between sensor and read out system which are very challenging to implement in a portable system where disposable cartridges are required to align with the read-out each time a new cartridge is inserted in the reader unit. Finally, we implemented a compact optical lever method with pick up fibers as explained in the previous sections. 
However, small mis-alignments degraded the SNR a lot. In this method, the cartridge and the reader unit should align better than $20 \mu \mathrm{m}$ which requires a complex active alignment of the cartridge inside the reader unit. However, our last approach, where we used a vibrating optical sensor, relaxed the alignment tolerances and we managed to implement this measurement approach in a prototype unit as shown in Fig. 5. We measured coagulation times with high reliability using the developed prototype. Optical fiber based sensor eased alignment tolerances by employing large core diameter multimode fibers. Since pick up fiber is embedded in the cartridge, only light into and out of cartridge needs to be coupled. Light is coupled from a LED source to vibrating fiber and pick up fiber to photo detector by butt-to-butt coupling. Simplicity of the optical read out scheme of fiber based sensor further encourages a low cost PoC application.
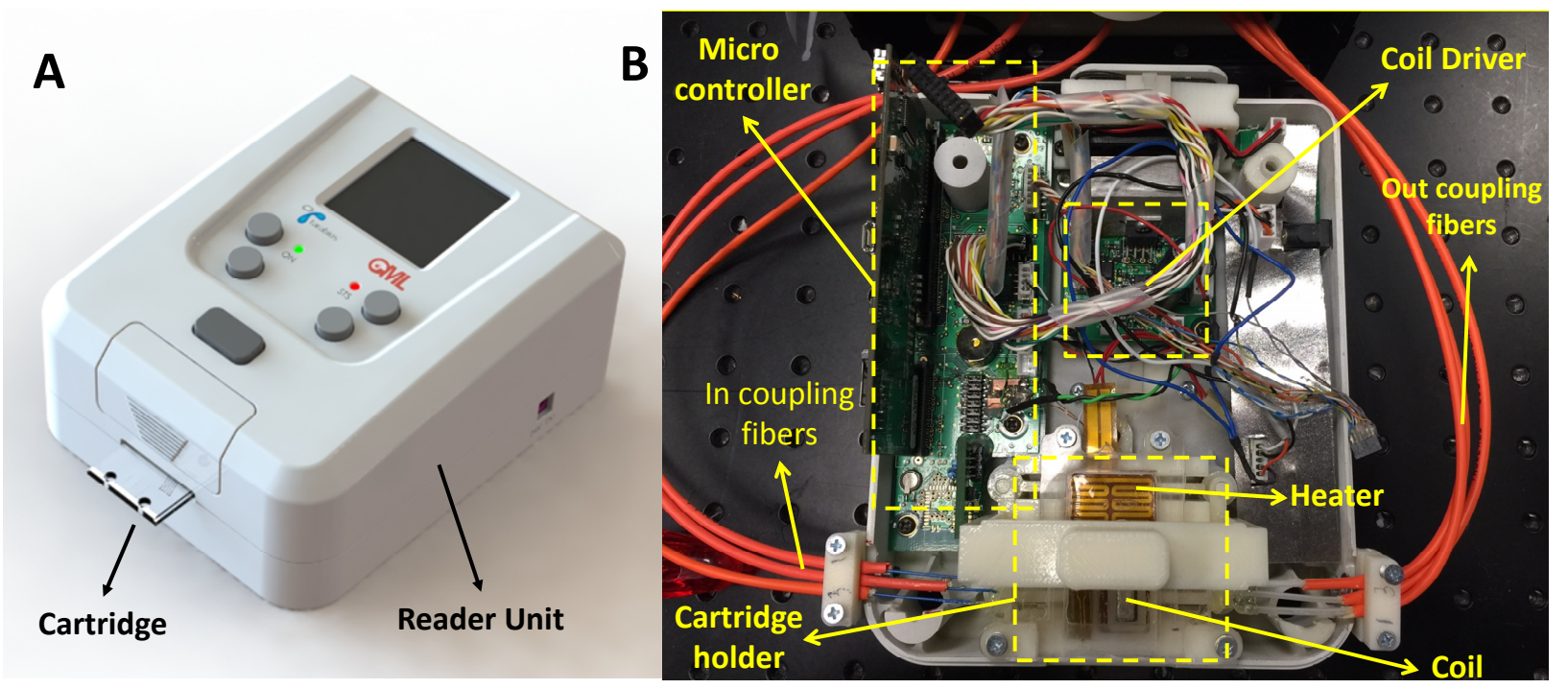

Figure 5. (A) Prototype . developed for the MEMS based cartridges (B) Inside the prototype unit, the unit is modified to add longer coupling fibers. Components of the prototype of the reader unit are also shown.

The prototype, as shown in Fig. 5, was developed with the help SFO Technologies, India. Lock-in-amplifier, signal generator and temperature control were implemented with a microcontroller. Amplifier stages were designed for driving the electromagnetic coil and signal amplification. User interface was provided with an LCD display and push buttons. Note that, this prototype was developed for simultaneous 3 channel operation. Size of the prototype can be further reduced for one channel operation with design optimizations.

\section{RESULTS}

\subsection{Sensor Characterization}

Sensors were characterized in various mediums before control plasma and whole blood tests. Sensors were tested in air, distilled water and various plasma samples. As viscosity of the medium increases, a decrease in resonance frequency and quality factor is observed due to hydrodynamic loading. Highest change is observed between air and distilled water due to high viscosity difference. Resonance characteristics of MEMS based sensor and optical fiber based sensor are shown in Fig. 6. MEMS based sensor is more sensitive to viscosity changes due to it is geometric shape and small spring constant. On the other hand, optical fiber based system is less sensitive to viscosity since circular shape of optical fiber has a small drag coefficient. Resonant frequency of the MEMS cantilever was dropped from $39206 \mathrm{~Hz}$ to $22185 \mathrm{~Hz}$ and quality factor was dropped from 178 to 4.5 whereas in optical fiber based sensor resonance frequency was dropped from $4703 \mathrm{~Hz}$ to 4300 and quality factor was dropped from 62 to 34 between air and distilled water mediums. In plasma tests, the phase of the MEMS sensor gave a clearer signal than its amplitude whereas amplitude of the optical fiber sensor provided a higher SNR signal compared to the signal obtained from its phase. 
A

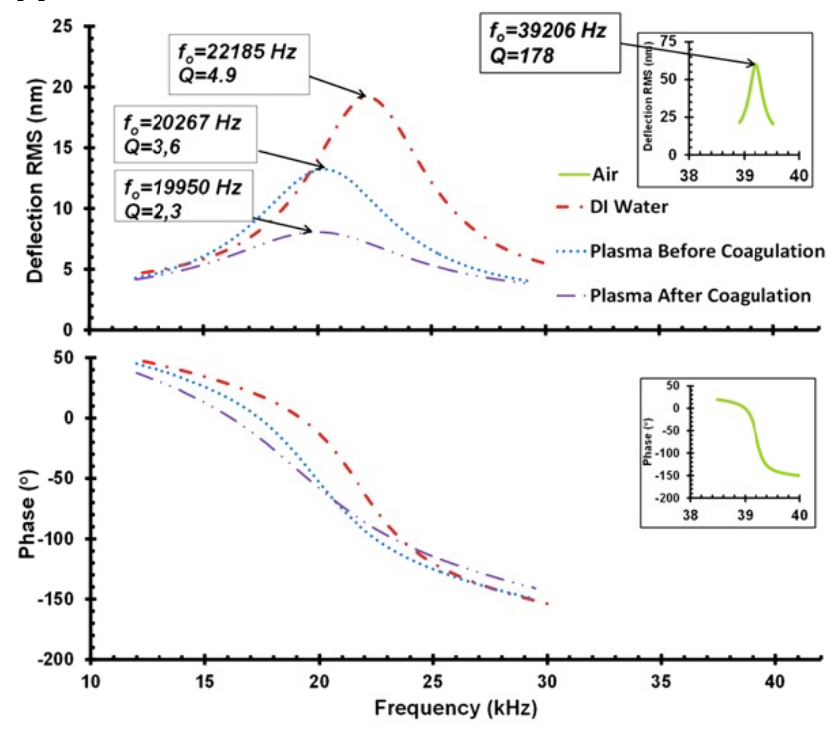

B

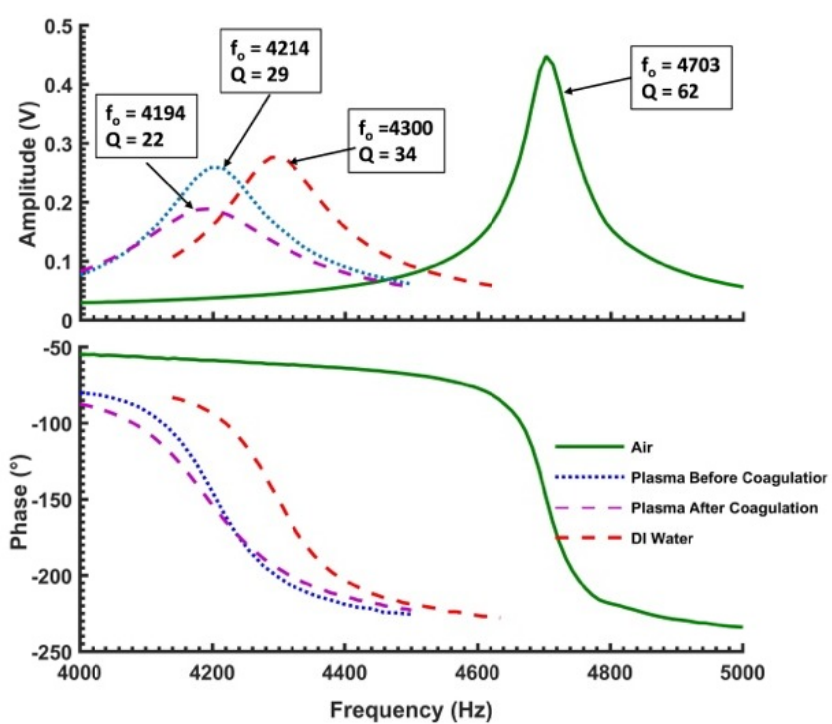

Figure 6. Frequency sweeps in different mediums for (A) MEMS based sensor ${ }^{12}$ and (B) optical fiber based sensor

\section{2. aPTT test results}

aPTT tests with plasma and whole blood samples were conducted using both MEMS based sensor and optical fiber based sensor. Three different control plasma samples were used; normal, abnormal 1, and abnormal 2 from DIAGEN, UK. Normal control plasma samples represent a healthy person's plasma whereas abnormal 1 and abnormal 2 represent longer coagulation times. The proposed biosensor platform was further tested with whole blood samples. Since MEMS based sensor suffered from low SNR, whole blood samples were tested with optical fiber based sensor only. Whole blood samples were collected from a 24 years old healthy male donor. Before measurements, all samples and reagents were warmed to $37^{\circ} \mathrm{C}$. $50 \mu \mathrm{L}$ of the sample was mixed with $50 \mu \mathrm{L}$ Micronised Silica/Platelet Substitute mixture (DIAGEN, UK) which is a common aPTT reagent used in coagulation tests. Sample-reagent mixture was incubated for exactly 5 minutes. After incubation, $50 \mu \mathrm{L}$ of $25 \mathrm{mM} \mathrm{CaCl}_{2}$ solution was mixed with the mixture and $12 \mu \mathrm{L}$ of this final mixture was inserted into the cartridge immediately. Since samples are citrated in order to prevent clot formation during storage, coagulation process does not start until the introduction of $\mathrm{CaCl}_{2}$. Therefore, mixing of $\mathrm{CaCl}_{2}$ solution into mixture is taken as $t=0$. Coagulation is tracked by amplitude and phase change in the read out signal. Fig. 7 shows the amplitude and phase change for MEMS based sensor during coagulation. Optical fiber based sensor has similar trend in amplitude and phase. In order to determine the exact time of the coagulation, a curve was fitted to the phase data for MEMS based sensor (amplitude data for fiber based sensor) and the first derivative of the curve was calculated. The local minima of the derivative is taken as the coagulation time ${ }^{16}$. Since every coagulation measurement platform uses different detection techniques and reagents, results are normalized within the particular measurement platform in order to compare the test results. We took the average of the normal plasma test results as the scaling factor. Table 1 compares the aPTT times for both MEMS based sensor and optical fiber based sensor and manufacturer's datasheet. Both sensors give concordant results with the results indicated in the datasheet of the control plasma samples. The variation of the fiber-based sensor's results are better compared to MEMS device. This can be related with the variations among the MEMS chips used in different experiments. Another important point is about the whole blood measurements, the results obtained with the fiber-based system has an CV \% in the acceptable limits of most of the commercial coagulameters. 

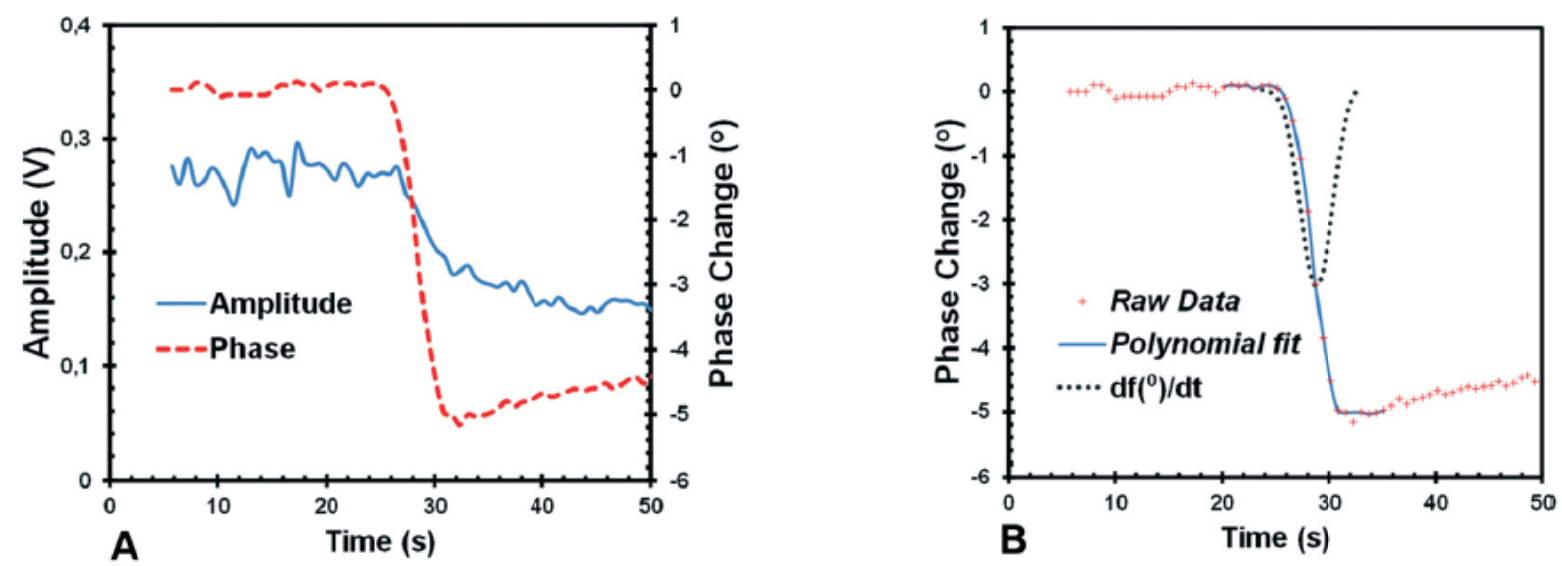

Figure 7. (A) The phase and amplitude during a PT test for an abnormal 1 control plasma sample. (B) Polynomial fit to the phase data (solid blue) and the first derivative of the fit function (dotted black); the local minima is chosen as the coagulation time. ${ }^{12}$

\begin{tabular}{|c|c|c|c|c|c|}
\hline & & $\begin{array}{l}\text { Normal } \\
\text { plasma }\end{array}$ & $\begin{array}{l}\text { Abnormal } 1 \\
\text { plasma }\end{array}$ & $\begin{array}{l}\text { Abnormal } 2 \\
\text { plasma }\end{array}$ & $\begin{array}{l}\text { Whole } \\
\text { blood }\end{array}$ \\
\hline \multirow{3}{*}{$\begin{array}{l}\text { MEMS } \\
\text { based } \\
\text { sensor }\end{array}$} & Normalized aPTT ratio & 1.01 & 1.19 & 1.78 & \\
\hline & $\sigma$ & 0.04 & 0.11 & 0.1 & \\
\hline & $\mathrm{CV} \%$ & 3.94 & 9.11 & 5.87 & \\
\hline \multirow{3}{*}{$\begin{array}{l}\text { Optical } \\
\text { fiber based } \\
\text { sensor }\end{array}$} & Normalized aPTT ratio & 1.03 & 1.45 & 2.22 & 1.05 \\
\hline & $\sigma$ & 0.014 & 0.007 & 0.012 & 0.021 \\
\hline & $\mathrm{CV} \%$ & 1.36 & 0.48 & 0.54 & 2 \\
\hline \multirow{3}{*}{ Datasheet } & Normalized aPTT ratio & 1.03 & 1.4 & 2.2 & \\
\hline & & 0.007 & 0.015 & 0.038 & \\
\hline & $\mathrm{CV} \%$ & 0.68 & 1.05 & 1.73 & \\
\hline
\end{tabular}

Table 1. Normalized aPTT test results with MEMS and Fiber-optic based sensors in comparison with the control plasma datasheet. $\mathrm{n}=9$ for MEMS based sensor whereas $\mathrm{n}=3$ for optical fiber based sensor.

\section{CONCLUSION}

A direct mechanical coagulation measurement platform is introduced with two different sensors: MEMS and optical fiber based sensors. Both sensors utilize remote electromagnetic actuation and optical read out. Platform was tested with plasma and whole blood samples. Test results are concordant with the values at the datasheet of the control plasma manufacturer's. The experiments show a CV \% below $10 \%$ for MEMS based system, whereas optical fiber based system results are below $2 \%$ for the aPTT tests. MEMS based sensors are difficult to implement in a portable unit where simplicity is very important for cost reasons. The fiber based cartridge approach solves the complex alignment problem. The fiber sensor based system is suitable for a PoC application due to cost effective cartridge and simple read out scheme. 


\section{ACKNOWLEDGEMENT}

This work is supported by TÜBİTAK $113 \mathrm{~S} 074$ grant. We thank Dr. Samuel Varghese and Ahamed Ameen and their teams at SFO Technologies for help with the prototype development.

\section{REFERENCES}

[1] Ng, V. L., "Liver Disease, Coagulation Testing, and Hemostasis," JOUR, Clin. Lab. Med. 29(2), 265282 (2009).

[2] Korte, W., Gabi, K., Rohner, M., Gahler, A., Szadkowski, C., Schnider, T. W., Lange, J.., Riesen, W., "Preoperative fibrin monomer measurement allows risk stratification for high intraoperative blood loss in elective surgery.," Journal Article, Thromb. Haemost. 94(1), 211-215, Germany (2005).

[3] Lin, S.-W., Chuang, Y.-C., Lin, Y.-S., Lei, H.-Y., Liu, H.-S.., Yeh, T.-M., "Dengue virus nonstructural protein NS1 binds to prothrombin/thrombin and inhibits prothrombin activation.," Journal Article, Research Support, Non-U.S. Gov’t, J. Infect. 64(3), 325-334, England (2012).

[4] Lison, S.., Spannagl, M., "Monitoring of direct anticoagulants," Wiener Medizinische Wochenschrift 161(3-4), 58-62 (2011).

[5] Ojito, J. W., Hannan, R. L., Burgos, M. M., Lim, H., Huynh, M., Velis, E., Arocha, M., Tirotta, C. F.., Burke, R. P., "Comparison of point-of-care activated clotting time systems utilized in a single pediatric institution.," J. Extra. Corpor. Technol. 44(1), 15-20 (2012).

[6] Jackson, C. M.., Esnouf, M. P., "Has the time arrived to replace the quick prothrombin time test for monitoring oral anticoagulant therapy?," Clin. Chem. 51(3), 483-485 (2005).

[7] Harris, L. F., Castro-López, V.., Killard, A. J., "Coagulation monitoring devices: Past, present, and future at the point of care," TrAC - Trends Anal. Chem. 50, 85-95, Elsevier Ltd (2013).

[8] Yaroslavsky, A. N., Yaroslavsky, I. V., Goldbach, T.., Schwarzmaier, H.-J., "Optical properties of blood in the near-infrared spectral range," 1996, 314-324.

[9] “CoaguChek XS PT Test ${ }^{\circledR} . ”,<$ http://coaguchek-usa.com/documents/coaguchek-xs-plus-packageinsert.pdf $>$ (10 January 2016$)$.

[10] Van Eysden, C. A.., Sader, J. E., "Frequency response of cantilever beams immersed in viscous fluids with applications to the atomic force microscope: Arbitrary mode order," J. Appl. Phys. 101(4) (2007).

[11] Chon, J. W. M., Mulvaney, P.., Sader, J. E., "Experimental validation of theoretical models for the frequency response of atomic force microscope cantilever beams immersed in fluids," J. Appl. Phys. 87(8), 3978-3988 (2000).

[12] Cakmak, O., Ermek, E., Kilinc, N., Bulut, S., Baris, I., Kavakli, I. H., Yaralioglu, G. G.., Urey, H., “A cartridge based sensor array platform for multiple coagulation measurements from plasma," Lab Chip 15(1), 113-120, Royal Society of Chemistry (2015).

[13] Cakmak, O., Ermek, E., Urey, H., Yaralioglu, G. G.., Kilinc, N., "MEMS based blood plasma viscosity sensor without electrical connections," IEEE SENSORS 2013 - Proc. (2013).

[14] Cakmak, O., Elbuken, C., Ermek, E., Mostafazadeh, A., Baris, I., Erdem Alaca, B., Kavakli, I. H.., Urey, H., "Microcantilever based disposable viscosity sensor for serum and blood plasma measurements," Methods 63(3), 225-232, Elsevier Inc. (2013). 
[15] Mostafazadeh, A., Yaralioglu, G. G., Urey, H., "Optical fiber array based simultaneous parallel monitoring of resonant cantilever sensors in liquid," Sensors Actuators, A Phys. 242, 132-139, Elsevier B.V. (2016).

[16] Doubleday, K., Kumnick, S., "Clot Signature Curves and the ACL Advance,” Instrum. Lab. (2001). 